\title{
Effects of time delays and viscoelastic parameters in oscillatory response cell monolayers
}

\author{
Cristian Borja ${ }^{1}$, Elena Moral ${ }^{2}$, Jose J. Muñoz ${ }^{3}$
}

1. Universitat de Barcelona, Spain

2. Universitat Politècnica de Catalunya, Barcelona, Spain. Currently at Technishe Universiteit München.

3. Laboratori de Càlcul Numèric (LaCàN). Universitat Politècnica de Catalunya, Barcelona, Spain j.munoz@upc.edu

\begin{abstract}
Oscillatory response of cellular tissues has been observed in multiple embryogenetic developmental stages. The source of these oscillations has been attributed to imbalance of instabilities in the chemo-mechanical signalling and delayed cell activity. Although the relation of these oscillations with further drastic tissue deformation remains uncertain, it is apparent that intracellular remodelling events seem to drive the viscoelastic properties and the measured pulsatile deformations.

We here resort to a viscoelastic model that is based on a variable restlength of the cell. We include a delay between the measured elastic strain and the evolution of the rest-length which dynamically adapts to the cell strain. This law is not only able to reproduce the relaxation phenomena observed in embryonic tissues in vitro and in vivo, but also to give rise to oscillatory cell responses. We analyse the stability of the resulting oscillations on minimal systems with two cells, and also on planar or out of plane deformation modes of monolayers. We conclude that in all cases, the stability decreases with an increasing delay or with the ability to adapt in a faster manner.
\end{abstract}

keywords: Oscillations, tissues, stability, delay differential equations, embryogenesis, Monolayers

\section{Introduction}

The analysis of the oscillatory behaviour of cells has attracted the attention of experimental and theoretical researchers in recent years. They are ubiquitous and precede drastic morphogenetic shape changes. For instance, sustained planar oscillations of monolayers have been observed during embryonic germband extension in Drosophila embryo [15], or during dorsal closure [24, 7] or ventral invagination [14].

These oscillations have been associated with the coupling between the chemical and mechanical signalling $[19,11]$, or the relation between cell polarity and force transmission $[18,21]$, with a potential dependence on the confinement [20]. More recently, it has been observed that the relocation of actomyosin components from medial to junctional areas of the cell may trigger these pulsatile response, suggesting that this biochemical delay between motor molecules is responsible of the observed oscillations [26]. 
Some recent works have analysed the dynamics of the chemo-mechanical coupling through diffusion reaction equations, with applications in apical constriction in epithelia [11], monolayers on substrates [18, 21] or wound healing [22]. In order to analyse the stability of such systems, we here focus on the delay and model it explicitly by resorting to a delayed cell rheology and the resulting delay differential equations, in similar manner to the stress analysis in yeast [13], neural axons conduction [3], transport in respiratory systems [5], or in cell maturation [10].

We here analyse the stability and oscillatory response of a planar deformation mode that preserves cell areas. We include a viscous like rheological law based on an adaptive rest-length [16], where the latter varies according to a delayed measure of strains. This work extends previous uni-dimensional analysis [17] to a planar and non-linear mode of deformation.

This chapter is structured as follows. We first present the periodic planar deformation mode and the resulting equilibrium equations for are preserving planar deformations of a tissue in Section 2. We then analyse the linearised equations and the resulting delay differential equations, and provide bounds for the delay and material parameters in Section 2.3. We also study the stability and oscillatory bounds of the non-linear system (Section 2.4. We also present the equilibrium equations for the cross-section of the tissue in Section 3, and analyse the stability for on-plane (Section 3.1) and out-of-plane (Section 3.2) deformations. We finally give some concluding remarks in Section 4.

\section{Planar deformations}

\subsection{Planar deformation mode and equilibrium equations}

We will simplify our mechanical analysis by assuming that the epithelium can be represented by a two-dimensional patch of regular hexagons, as shown in Figure 1a. We will additionally suppose that the cells deform in an area preserving mode, and that this deformation has the same periodicity of the whole pattern. The tissue deformation is thus fully described by the vertex displacement given in the greyed area of Figure 1b. Although this periodicity is not exactly observed experimentally, cells locally share similar distortions. The simplification employed here also allows us to parametrise the whole deformation through the displacement of a single vertex, denoted by $\boldsymbol{u}=\left\{\begin{array}{ll}u_{x} & u_{y}\end{array}\right\}^{T}$, and to obtain analytical results on the stability of the resulting oscillations.

According to these assumptions, the planar deformations of the two-dimensional patch are fully described with the 3 elements depicted in Figure 1b, which represent the junctions of three generic cells. We will henceforth study the motion of the cells in the system by analysing the equilibrium equations and the rheology of these 3 elements.

The equilibrium equations of the 3 element system are written in terms of 


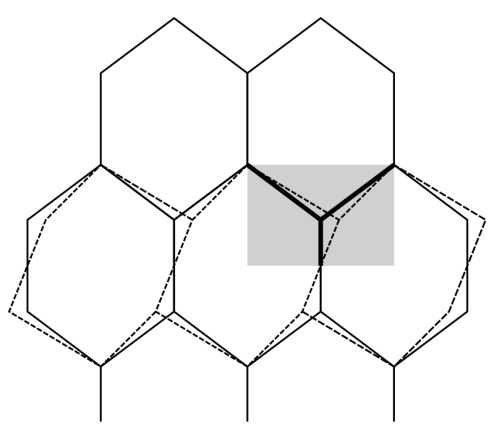

(a)

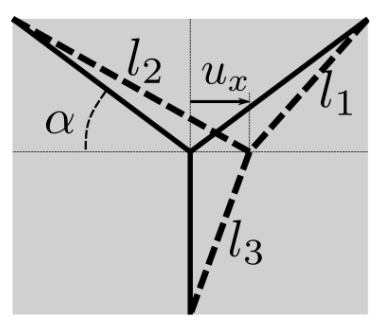

(b)

Figure 1: (a): scheme of distribution of cells as a regular pattern of hexagons. (b): detail of the vertex displacement in the shaded are of the left.

an elastic potential function $W(\boldsymbol{u})$ given by,

$$
W(\boldsymbol{u})=\frac{1}{2} \sum_{i=1}^{3} k\left(l_{i}(\boldsymbol{u})-L_{i}\right)^{2}
$$

where $k$ is the element stiffness, assumed as constant and equal for all the elements, $l_{i}(\boldsymbol{u})$ is the apparent (observed) length of each element $i=1,2,3$, and $L_{i}$ its rest-length. We point out that the latter is assumed to be not necessarily constant. Its evolution will be detailed in the next section.

The equilibrium equations are deduced from the stationary condition of $W(\boldsymbol{u})$ as,

$$
\left.\frac{d W(\boldsymbol{u})}{d \boldsymbol{u}}\right|_{L_{i}=\text { const }}=\mathbf{0}
$$

which from the expression of $W(\boldsymbol{u})$ in (1) yields,

$$
\sum_{i=1}^{3}\left(l_{i}-L_{i}\right) \frac{d l_{i}}{d \boldsymbol{u}}=\mathbf{0}
$$

Note that the equation above includes two scalar equations, one for $u_{x}$ (horizontal equilibrium) and another for $u_{y}$ (vertical equilibrium). For simplicity, we will assume that $u_{y}=0$, and denote by $u$ the horizontal displacement $u_{x}$. The general case with $u_{y} \neq 0$ will be discussed in Section 2.4, and in fact, a very similar analysis to the one presented here can be deduced when $u_{y}$ is not zero. 
The expressions of $l_{i}$ in terms of $u$ are found using elemental geometry,

$$
\begin{aligned}
& l_{1}=\sqrt{\left(l_{0} \sin \alpha\right)^{2}+\left(l_{0} \cos \alpha-u\right)^{2}} \\
& l_{2}=\sqrt{\left(l_{0} \sin \alpha\right)^{2}+\left(l_{0} \cos \alpha+u\right)^{2}} \\
& l_{3}=\sqrt{l_{0}^{2}+u^{2}}
\end{aligned}
$$

where $\alpha$ is the initial angle that elements 1 and 3 form with the horizontal axis, and $l_{0}$ is the length of the undeformed configuration, assumed equal for all the elements (see Figure 1b). Using the expressions above, the horizontal mechanical equilibrium is deduced from (2) as,

$$
\left(l_{1}-L_{1}\right) \frac{u-l_{0} \cos \alpha}{l_{1}}+\left(l_{2}-L_{2}\right) \frac{l_{0} \cos \alpha+u}{l_{2}}+\left(l_{3}-L_{3}\right) \frac{u}{l_{3}}=0
$$

In the subsequent sections, we will use the compact notation $\boldsymbol{L}(t)=\left\{L_{1}(t) L_{2}(t) L_{3}(t)\right\}^{T}$ as the vector of unknown rest-lengths. Consequently, by replacing the geometrical relations in (3)-(5) into the previous non-linear equation, it can be re-casted as

$$
G(u, \boldsymbol{L})=0
$$

\subsection{Cortex rheology and DDEs}

We assume that each element has the ability to adapt to its current length $l_{i}$ by changing its resting length $L_{i}$ according to the following evolution law,

$$
\dot{L}_{i}(t)=\gamma_{i}\left(l_{i}(t-\tau)-L_{i}(t-\tau)\right), i=1,2,3
$$

where $\gamma_{i}>0$ is a material parameter called the remodelling rate, and $\tau>0$ is a delay that measures the retarding time between the measured strain $l_{i}-L_{i}$ and the cell active rest-length changes. Parameters $\gamma_{i}$ represents the rate at which cells adapt their length. It has been proved that when $\tau=0$, the evolution law in (8) mimics Maxwell viscoelastic response [16]. Such model has been also employed in embryogenesis $[2,4,8,25]$ or relaxation tests on suspended monolayers [12].

The presence of delay $\tau$ is motivated by the observed oscillatory response and the measured delay between myosin concentration and area oscillation in Drosophila fly dorsal closure [7]. The stability of single elements with the evolution law in (8) has been analysed in [17]. We are here interested in extending these results to multicellular systems.

The presence of the the delay $\tau$ in (8) turns this differential equation into a socalled Delay Differential Equation (DDE) [9], which jointly with the equilibrium equation in (6) and the geometrical relations in (3)-(5) will allow to analyse the stability of our primary unknowns $u(t)$ and $L_{i}(t), i=1,2,3$. More precisely, by 
replacing the expression of $l_{i}$ in (8) by the relations in (3)-(5), the time evolution of $u$ and $\boldsymbol{L}(t)$ are given by the following system of algebraic-DDE,

$$
\left\{\begin{array}{l}
\dot{\boldsymbol{L}}(t)=\boldsymbol{f}(u(t-\tau), \boldsymbol{L}(t-\tau)) \\
G(u(t), \boldsymbol{L}(t))=0
\end{array}\right.
$$

with the initial conditions

$$
\left.\begin{array}{rl}
\boldsymbol{L}(t) & =\boldsymbol{L}_{0} \\
u(t) & =u_{0}
\end{array}\right\} \forall t \in[-\tau, 0] .
$$

The explicit expression of function $\boldsymbol{f}(u(t), \boldsymbol{L}(t))$ in $(9)$ is

$$
\boldsymbol{f}(u(t), \boldsymbol{L}(t))=\left\{\begin{array}{c}
\gamma_{1}\left(\sqrt{\left(l_{0} \sin \alpha\right)^{2}+\left(l_{0} \cos \alpha-u(t)\right)^{2}}-L_{1}(t)\right) \\
\gamma_{2}\left(\sqrt{\left(l_{0} \sin \alpha\right)^{2}+\left(l_{0} \cos \alpha+u(t)\right)^{2}}-L_{2}(t)\right) \\
\gamma_{3}\left(\sqrt{l_{0}^{2}+u(t)^{2}}-L_{3}(t)\right)
\end{array}\right\} .
$$

Note that due to the presence of the DDE in (9), the initial conditions are defined on the interval $[-\tau, 0]$, and that we have assumed for simplicity that $u_{0}$ and $\boldsymbol{L}_{0}=\left\{\begin{array}{llll}L_{10} & L_{20} & L_{30}\end{array}\right\}^{T}$ are constant. For consistency, the values of $u_{0}$ and $\boldsymbol{L}(0)$ should satisfy $G\left(u_{0}, \boldsymbol{L}_{0}\right)=0$. We will next study the linearised form of the initial value problem in (9)-(11).

\subsection{Linearised equations of planar deformation}

\subsubsection{Linear delay-differential equations}

The algebraic-DDE system is non-linear due to the expression of $\boldsymbol{f}(u(t), \boldsymbol{L}(t))$ and $G(u(t), \boldsymbol{L}(t))$. We will henceforth assume that $u(t) \ll 1$, and that the apparent lengths $l_{i}(t)$ can be approximated by the linear terms,

$$
\begin{aligned}
& l_{1}(t) \approx l_{0}-u(t) \cos \alpha, \\
& l_{2}(t) \approx l_{0}+u(t) \cos \alpha, \\
& l_{3}(t) \approx l_{0},
\end{aligned}
$$

which are valid at all times. By inserting this linearised forms into the DDEs in (8) we obtain,

$$
\left\{\begin{array}{l}
\dot{L}_{1}(t)=-\gamma_{1} u(t-\tau) \cos \alpha-\gamma_{1} L_{1}(t-\tau)+\gamma_{1} l_{0} \\
\dot{L}_{2}(t)=\gamma_{2} u(t-\tau) \cos \alpha-\gamma_{2} L_{2}(t-\tau)+\gamma_{2} l_{0} \\
\dot{L}_{3}(t)=-\gamma_{3} L_{3}(t-\tau)+\gamma_{3} l_{0}
\end{array}\right.
$$

Furthermore, the linearised form of the equilibrium equation in $(6)$ at $(u, \boldsymbol{L})=$ $\left(0, \boldsymbol{L}_{0}\right)$ becomes:

$$
\left(3-\frac{L_{10}}{l_{0}} \sin ^{2} \alpha-\frac{L_{20}}{l_{0}} \sin ^{2} \alpha-\frac{L_{30}}{l_{0}}\right) u(t)+\cos \alpha\left(L_{1}(t)-L_{2}(t)\right)=0
$$


which after setting

$$
\lambda_{0}=3-\frac{L_{10}}{l_{0}} \sin ^{2} \alpha-\frac{L_{20}}{l_{0}} \sin ^{2} \alpha-\frac{L_{30}}{l_{0}}
$$

may be written at time $t-\tau$ as,

$$
u(t-\tau)=\frac{\cos \alpha}{\lambda_{0}}\left(L_{2}(t-\tau)-L_{1}(t-\tau)\right) .
$$

Replacing this expression of $u(t)$ into (13), our system of algebraic-DDE in (9)-(11) is approximated by the following linear system of DDEs:

$$
\left\{\begin{aligned}
\dot{\boldsymbol{L}}(t) & =\mathbf{A} \boldsymbol{L}(t-\tau)+l_{0} \boldsymbol{\gamma}, \\
\boldsymbol{L}(t) & =\boldsymbol{L}_{0},
\end{aligned} \forall t \in[-\tau, 0]\right.
$$

with

$$
\mathbf{A}=\left[\begin{array}{ccc}
\gamma_{1}\left(\frac{\cos ^{2} \alpha}{\lambda_{0}}-1\right) & -\gamma_{1} \frac{\cos ^{2} \alpha}{\lambda_{0}} & 0 \\
-\gamma_{2} \frac{\cos ^{2} \alpha}{\lambda_{0}} & \gamma_{2}\left(\frac{\cos ^{2} \alpha}{\lambda_{0}}-1\right) & 0 \\
0 & 0 & -\gamma_{3}
\end{array}\right], \gamma=\left\{\begin{array}{l}
\gamma_{1} \\
\gamma_{2} \\
\gamma_{3}
\end{array}\right\}
$$

\subsubsection{Solution of linear DDE system}

For simplicity we will study the stability of the DDE systems in (16)-(17) assuming that

$$
\gamma_{1}=\gamma_{2}=\gamma_{3}=\gamma
$$

In this case, since matrix $\mathbf{A}$ is a real symmetric matrix, it accepts real eigenvalues $\lambda_{1}, \lambda_{2}, \lambda_{3}$. Its eigenvalue decomposition may then be written as,

$$
\mathbf{A}=\mathbf{V}^{T} \mathbf{D V}
$$

with $\mathbf{D}=\operatorname{diag}\left(\lambda_{1}, \lambda_{2}, \lambda_{3}\right)$ and $\mathbf{V}=\left[\boldsymbol{v}_{1}, \boldsymbol{v}_{2}, \boldsymbol{v}_{3}\right]$ the matrix of normalised eigenvectors such that $\mathbf{V}^{T} \mathbf{V}=\mathbf{I}$. In our case, from the expression of $\mathbf{A}$ in (17) we have that,

$$
\mathbf{D}=\left[\begin{array}{rrc}
-\gamma & 0 & 0 \\
0 & -\gamma & 0 \\
0 & 0 & -\gamma\left(1-\frac{2 \cos ^{2} \alpha}{\lambda_{0}}\right)
\end{array}\right], \mathbf{V}=\frac{1}{\sqrt{2}}\left[\begin{array}{rrr}
1 & 0 & 1 \\
1 & 0 & -1 \\
0 & \sqrt{2} & 0
\end{array}\right]
$$

It follows that the DDE system in (16)-(17) is equivalent to the following diagonal system,

$$
\left\{\begin{array}{ll}
\dot{\boldsymbol{y}}(t) & =\mathbf{D} \boldsymbol{y}(t-\tau)+l_{0} \gamma \boldsymbol{v}, \\
\boldsymbol{y}(t) & =\mathbf{V} \boldsymbol{L}_{0},
\end{array} \quad \forall t \in[-\tau, 0]\right.
$$


with $\boldsymbol{y}(t)=\mathbf{V} \boldsymbol{L}(t)$ and $\boldsymbol{v}=\sum_{i} \boldsymbol{v}_{i}$. It is worth pointing out though that the linear system in (16) can be also written as,

$$
\left\{\begin{array}{cl}
\dot{L}_{2}(t)-\dot{L}_{1}(t) & =\gamma\left(1-\frac{2 \cos ^{2} \alpha}{\lambda_{0}}\right)\left(L_{2}(t-\tau)-L_{1}(t-\tau)\right) \\
\dot{L}_{3}(t) & =-\gamma L_{3}(t-\tau)+\gamma l_{0}
\end{array}\right.
$$

with initial conditions

$$
\left\{\begin{aligned}
L_{2}(t)-L_{1}(t) & =L_{20}-L_{10}, & & t \in[-\tau, 0] \\
L_{3}(t) & =L_{30}, & & t \in[-\tau, 0]
\end{aligned}\right.
$$

Remark 2.1 The latter forms prompts us to consider the following particular situation:

a) When $L_{10}=L_{20}$, due to the first $D D E$ in (22), we have that

$$
L_{2}(t)-L_{1}(t)=0 \quad \forall t \in[-\tau, \infty)
$$

This corresponds to reducing the dynamics to solely mode $\boldsymbol{v}_{3}$ in (20). Also, due to the equilibrium condition in (15), and when $\lambda_{0} \neq 0$, the displacement $u(t) \equiv 0$. In this case, the delay equations in (13) are simplified, and matrix A reduces to

$$
\mathbf{A}=-\gamma \mathbf{I}
$$

That is, $\lambda_{1}=\lambda_{2}=\lambda_{3}=-\gamma$.

b) Additionally, let us assume that $L_{10}=L_{20}=L_{30}$. Then, due to the form of the system matrix $\mathbf{A}$ in (23) and initial conditions, all rest-lengtsh have the same evolution:

$$
L_{1}(t)=L_{2}(t)=L_{3}(t) \quad \forall t \in[-\tau, \infty) .
$$

In order to analyse the stability of the DDE, let us introduce the general form of the solutions to the DDE:

Proposition 2.1 The solution of the DDE in (21) is given by function $\boldsymbol{y}_{m}=$ $\left\{y_{m 1}, y_{m 2}, y_{m 3}\right\}^{T}$, with components,

$$
y_{m i}(t)=e^{m_{i} t} y_{0 i}-\frac{l_{0} \gamma}{\lambda_{i}}, i=1,2,3,
$$

if $m_{i}$ is solution of the characteristic equations,

$$
\tau \lambda_{i}=m_{i} e^{m_{i}}, i=1,2,3
$$


The proposition can be proved by simply inserting the expression in (24) into (21), which gives rise to the condition

$$
\lambda_{i}=m_{i} e^{m_{i} \tau}, i=1,2,3 .
$$

By using the change of variable $t^{\prime}=t / \tau$, the DDE in (21) reads $d \boldsymbol{y} / d t^{\prime}=$ $\tau \mathbf{D} \boldsymbol{y}\left(t^{\prime}-1\right)-l_{0} \gamma \boldsymbol{v}$, and thus condition in (26) turns into condition in (25). The latter equation can be written in terms of the Lambert function $W(z)$, which is defined as the inverse function such that $z=W\left(z e^{z}\right), \forall z \in \mathbb{C}$, or equivalently, the solution of $[6,9,23]$,

$$
z=W(z) e^{W(z)} .
$$

Alternatively, functions $y_{m i}(t)$ in (24) are solution of the DDE if

$$
m_{i}=W\left(\lambda_{i} \tau\right), i=1,2,3 .
$$

Remark 2.2 Lambert function can be extended to matrices by using the definition [1, 27],

$$
\mathbf{W}(\mathbf{A}) e^{\mathbf{W}(\mathbf{A})}=\mathbf{A}
$$

In our case though, since $\mathbf{A}$ admits the diagonalisation $\mathbf{A}=\mathbf{V D V}^{T}$, the Lambert matrix expression reads $\mathbf{W}(\mathbf{A})=\mathbf{V}^{T} \mathbf{W}(\mathbf{D}) \mathbf{V}^{T}$, and thus the analysis of the values of $\mathbf{W}(\mathbf{A})$ reduces to the simpler scalar definition in (27).

Lambert function is multivalued, giving rise to a set of solutions (or branches) $m_{i k}, k \in \mathbb{Z}$ that satisfy equation (27) [6]. Due to the linearity of the DDE, component $y_{i}(t)$ of the general solution $\boldsymbol{y}(t)$ can be written as

$$
y_{i}(t)=\sum_{k=-\infty}^{+\infty} e^{m_{i k} t} c_{i k}-l_{0} \frac{\gamma}{\lambda_{i}}, i=1,2,3
$$

where the coefficients $c_{i k}, i=1,2,3, k \in \mathbb{Z}$ depend on the initial conditions.

\subsubsection{Stability and oscillatory analysis of linear DDE}

We first recall standard results of the Lambert function and the characteristic equation $[23,9]$ :

Proposition 2.2 Lambert function $W(z)$ satisfies,

$$
\begin{aligned}
\text { i) } \operatorname{Re}(W(z))>0 & \text { if } \operatorname{Re}(z)<-\frac{\pi}{2} \text { or } \operatorname{Re}(z)>0 \\
\text { ii) } \operatorname{Im}(W(z)) \neq 0 & \text { if } \operatorname{Re}(z)<-\frac{1}{e}
\end{aligned}
$$


The bounds in Proposition 2.2 can be verified by searching the values for which the real part or the imaginary part of $m e^{m}$ vanish [9]. Indeed, setting $m=a+i b$ and $z=\xi+i \eta$, equation $z=m e^{m}$ implies

$$
\begin{aligned}
& \xi=e^{a}(a \cos b-b \sin b) \\
& \eta=e^{a}(b \cos b+a \sin b) .
\end{aligned}
$$

When $a=0$, we have from the first equation that $\xi=b=\pi / 2 \pm k \pi, k \in \mathbb{Z}$. When $b \rightarrow 0$, the second equation gives $a=-\lim _{b \rightarrow 0} b \cot b=-1$, which inserted into the first equation yields $\xi=-1 / e$.

Since the solutions $y_{m i}(t)$ contain the term $e^{m_{i} t}$, we know that stable solution will be obtained whenever $\operatorname{Re}\left(m_{i}\right)<0$, and oscillations will appear whenever $\operatorname{Im}\left(m_{i}\right) \neq 0$. Therefore, from Proposition 2.2 and the eigenvalues $\lambda_{i}$ in (20), we conclude our main result for the linearised problem:

Proposition 2.3 The DDE in (16)-(17), with $\gamma_{1}=\gamma_{2}=\gamma_{3}=\gamma>0$ and $\tau>0$ will have unstable solutions if

ia) $L_{10} \neq L_{20}$ :

$$
\gamma \tau \max \left(1,1-\frac{2 \cos ^{2} \alpha}{\lambda_{0}}\right)>\frac{\pi}{2}, \text { or, } 1-\frac{2 \cos ^{2} \alpha}{\lambda_{0}}<0,
$$

ib) $L_{10}=L_{20}$ :

$$
\gamma \tau>\frac{\pi}{2}
$$

and oscillatory solutions if

iia) $L_{10} \neq L_{20}$ :

$$
\gamma \tau \max \left(1,1-\frac{2 \cos ^{2} \alpha}{\lambda_{0}}\right)>\frac{1}{e}
$$

iib) $L_{10}=L_{20}$ :

$$
\gamma \tau>\frac{1}{e}
$$

Consequently, we have that when $\lambda_{0}<0$ and $L_{10} \neq L_{20}$, the initial conditions $\boldsymbol{L}_{0}$ may determine the presence of oscillations, and that when $\lambda_{0}<\lambda_{\text {crit }}$ with

$$
\lambda_{\text {crit }}=2 \cos ^{2} \alpha,
$$

and $L_{10} \neq L_{20}$, the stability conditions depend also on the initial conditions. For instance, for $\alpha=\pi / 6$, and setting $L_{10} \approx L_{20}=L_{30}=m_{L} l_{0}$, with $m_{L} \geq 0$ a model constant, we have from (14) that $\lambda_{0} \approx 3-2 m_{L}$. Therefore, the initial conditions determine the stability of the DDE whenever $3-3 m_{L} / 2<2 \cos ^{2} \alpha=$ $3 / 2$, that is, when

$$
m_{L}>1 .
$$


This corresponds to compressive initial states $\left(L_{i 0}>l_{0}\right)$, which in cellular materials is not a common situation. When $\lambda_{0}>\lambda_{\text {crit }}$, the stability is determined by the value of $\gamma \tau$ because $0<\left(1-\frac{2 \cos ^{2} \alpha}{\lambda_{0}}\right)<1$, and for unstressed initial conditions $\left(L_{i 0}=l_{0}\right), \lambda_{0}=\lambda_{\text {crit }}$, and thus the stability is not affected by the initial conditions. A similar conclusion can be drawn when $L_{i 0}=0$ (bars are stretched to their maximum), which yields $\lambda_{0}=3$.

Figure 2 shows the stability diagram on the $(\gamma, \tau)$ plane for $L_{10}=L_{20}$, which shows the same patterns as the one for one-element system [17]. Figure 3 illustrates the dependence of the stability on $\lambda_{0}$. The Figure shows the analytical results for the linear system (a) and also the numerical results for the non-linear system (b). The time evolution of $\boldsymbol{L}(t)$ is also shown for a value of $\lambda_{0}$ in Figure 4. The fact that the non-linear system shows no dependence on the initical conditions has motivated the analysis of the following section.

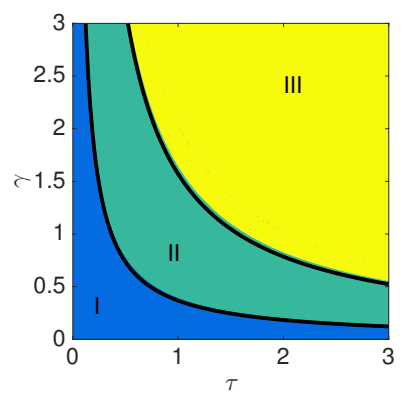

Figure 2: Stability diagram on the $(\gamma, \tau)$ plane for $L_{10}=L_{20}=L_{30}=0.9 l_{0}$, $\alpha=\pi / 6$, so that $\lambda_{0}=1.65$.

\subsection{Non-Linear system of planar deformations}

We have numerically solved the non-linear system in (9) for $\gamma_{1}=\gamma_{2}=\gamma_{3}=\gamma$, and verified that it exhibits no dependence on the initial conditions. The same stability diagram shown in Figure 2 is obtained for all values of $\boldsymbol{L}_{0}$. This fact will be justified in the next result:

Proposition 2.4 The system of non-linear DDE in (9) admits the solution $\dot{u}=0$ if $\gamma_{1}=\gamma_{2}=\gamma_{3}=\gamma$ and the initial conditions preserve equilibrium, i.e. $\boldsymbol{G}\left(u_{0}, \boldsymbol{L}_{0}\right)=\mathbf{0}$.

Proof 2.1 This can be proved by verifying that indeed the solution $\dot{u}=0$ is compatible with the equilibrium balance in (2) and the associated DDE equations in (8). First note that when $u=$ const, we have that $d l_{i} / d u=c_{i}=$ const and $l_{i}(t) \equiv l_{0}$. Therefore, the equilibrium equations

$$
\sum_{i=1}^{3} \frac{d W}{d l_{i}} \frac{d l_{i}}{d u}=0
$$



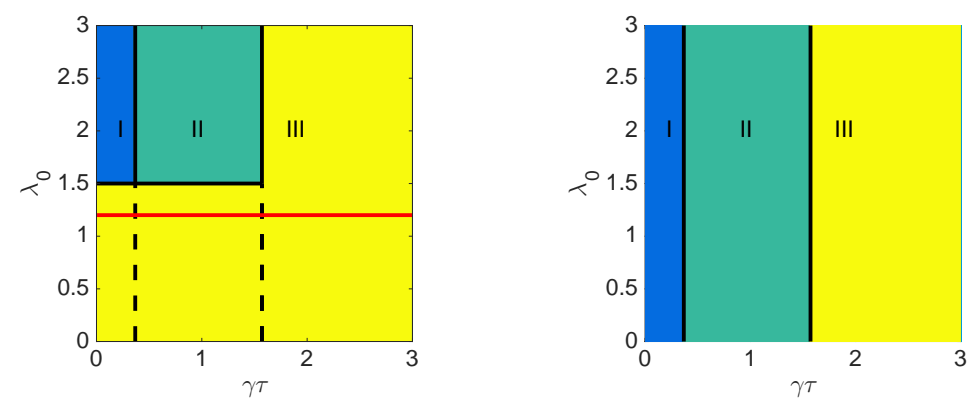

Figure 3: Stability diagram of the linear system (a) and non-linear system (b) in the $\left(\lambda_{0}, \gamma \tau\right)$ plane, obtained with $l_{0}=1, \alpha=\frac{\pi}{6}, L_{10}=L_{30}=1.2 l_{0}$ and $L_{20}$ calculated with the expression of $\lambda_{0}$ in (14). The vertical black lines are the critical values of the stability and oscillatory: $\frac{1}{e}$ and $\frac{\pi}{2}$, respectively. The horizontal black line is the value of $\lambda_{\text {crit }}=2 \cos ^{2} \alpha$. The red line is the value of $\lambda_{0}$ such that $L_{10}=L_{20}$ and corresponds to the value $\lambda_{0}=3-\frac{L_{20}}{l_{0}}\left(2 \sin ^{2} \alpha+1\right)$. The sectors: I are the stable and non-oscillatory solutions, II are the stable and oscillatory solutions and III are the unstable solutions.

read

$$
\sum_{i=1}^{3}\left(l_{0}-L_{i}(t)\right) c_{i}=0
$$

The time variation of this equation yields,

$$
-\sum_{i=1}^{3} \dot{L}_{i}(t) c_{i}=0
$$

which must hold $\forall t \in[-\tau, \infty)$. By replacing $\dot{L}_{i}(t)$ by the expression of the three evolution laws in (8) we obtain an equivalent equation to the equilibrium in (33),

$$
\gamma \sum_{i=1}^{3}\left(l_{0}-L_{i}(t-\tau)\right) c_{i}=0 .
$$

If we denote by $G(u(t), \boldsymbol{L}(t))=0$ the equilibrium equation in (33), we have thus shown that when $\dot{u}=0$ and $\gamma_{1}=\gamma_{2}=\gamma_{3}$, the evolution laws in (8) imply that,

$$
-\frac{d}{d t} G\left(u_{0}, \boldsymbol{L}(t)\right)=\gamma G\left(u_{0}, \boldsymbol{L}(t-\tau)\right)
$$

Consequently, if the initial conditions are such that $G\left(u_{0}, \boldsymbol{L}_{0}\right)=0$, i.e. equilibrium is preserved during $t \in[-\tau, 0]$, then equilibrium is preserved at all times due to the evolution laws. 


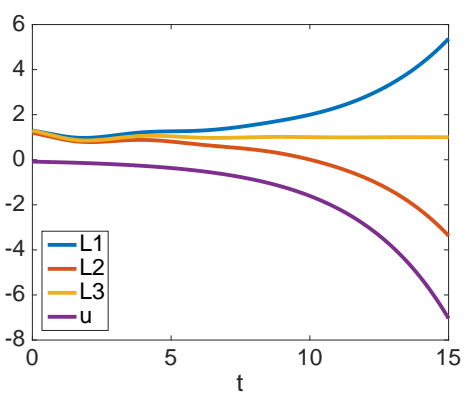

(a)

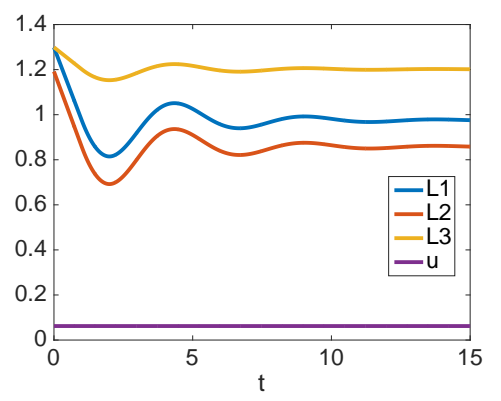

(b)

Figure 4: Numerical simulations with $l_{0}=\gamma=\tau=1, \alpha=\frac{\pi}{6}, L_{10}=L_{30}=1.3 l_{0}$, $L_{20}=1.2 l_{0}, \lambda_{0}=1.075$. (a): Linear problem. (b): Non-linear problem.

The stability bounds in the non-linear case can now be explained by pointing out that when $\dot{u}=0$, each element has a constant length $l_{i}=l_{0}$, and therefore behaves as a single element, where the evolution laws in (8) become independent and linear. The stability can be then analysed like in the unidimensional case [17], with stability bounds for $\gamma \tau$ given by $\pi / 2$.

Remark 2.3 The result of Proposition 2.4 for the non-linear systems also holds if vertical displacements are allowed, i.e. $u_{y} \neq 0$. In this case, when $\dot{\boldsymbol{u}}=0$, the equilibrium equations are written for components $x$ and $y$ as,

$$
\sum_{i=1}^{3}\left(l_{0}-L_{i}(t)\right) \boldsymbol{c}_{i}=\mathbf{0}
$$

with $\boldsymbol{c}_{i}=\frac{d l}{d \boldsymbol{u}}=\left\{d l_{i} / d u_{x} d l_{i} / d u_{y}\right\}^{T}$. If this equation is expressed as $\boldsymbol{G}\left(\boldsymbol{u}_{0}, \boldsymbol{L}(t)\right)=$ $\mathbf{0}$, a similar relation to the one in (36) is derived, and thus the same conclusions apply.

Remark 2.4 Proposition 2.4 does not necessary hold when the parameters $\gamma_{i}$ are not equal, or for the linear system. Indeed, the linearised equilibrium equations at a point $u=0, L_{i}=L_{i 0}$ are derived from the expression

$$
G\left(0, \boldsymbol{L}_{0}\right)+\left.\frac{d G(u, \boldsymbol{L})}{d u}\right|_{\left(0, \boldsymbol{L}_{0}\right)} u+\left.\sum_{i=1}^{3} \frac{d G(u, \boldsymbol{L})}{d L_{i}}\right|_{\left(0, \boldsymbol{L}_{0}\right)}\left(L_{i}-L_{i 0}\right)=0
$$

which result into the equation written in (15),

$$
\lambda_{0} u(t)+\left(L_{1}(t)-L_{2}(t)\right) \cos \alpha=0,
$$

with $\lambda_{0}$ given in (14). This equation, which is the linearised version of (32), differs from the form in (33) and may not be compatible with the evolution laws in (8). In fact, the time differentiation of (37) reveals that if $\dot{u}=0$, 
then $\dot{L}_{1}=\dot{L}_{2}$. However, the evolution laws in (8) imply that $\dot{L}_{1} \neq \dot{L}_{2}$ when $L_{10} \neq L_{20}$. Consequently, the solution $\dot{u}=0$ is not admissible for this initial condition.

\section{Analysis of tissue cross-section}

\subsection{On-plane deformation}

We consider here a strip of tissue where cells are adjacent to each other and a horizontal displacement $\boldsymbol{u}(t)$ acting on the nodes. We will first analyse onplane displacements that preserve cross-section area and that combine top and bottom surface, as indicated in Figure 6 .

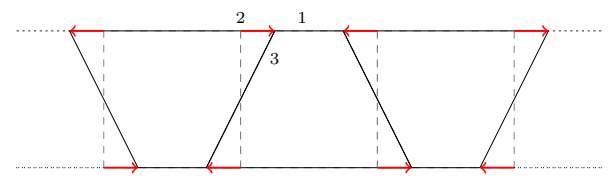

Figure 5: A section of tissue under the displacement $\boldsymbol{u}(t)$-in red-. Edges are enumerated.

By additionally considering a constant height assumption, the DDE for the rest-length and the equilibrium condition lead to a fully determined system,

$$
\left\{\begin{array}{l}
\dot{L_{1}}(t)=\gamma\left(l_{0}-2 u(t-\tau)-L_{1}(t-\tau)\right) \\
\dot{L_{2}}(t)=\gamma\left(l_{0}+2 u(t-\tau)-L_{2}(t-\tau)\right) \\
\dot{L_{3}}(t)=\gamma\left(\sqrt{l_{0}^{2}+4 u^{2}(t-\tau)}-L_{3}(t-\tau)\right) \\
0=L_{2}(t)-L_{1}(t)-6 u(t)+\frac{2 u(t) L_{3}(t)}{\sqrt{l_{0}^{2}+4 u^{2}(t)}}
\end{array}\right.
$$

Let us consider $m_{u}$ and $m_{L}$ two dimensionless parameters such that:

$$
L_{1}(0)=L_{3}(0)=m_{L} l_{0}, \quad u(0)=m_{u} l_{0}
$$

In order to guarantee the initial equilibrium, we introduce a small perturbation $\epsilon$ such that $L_{2}(0)=m_{L} l_{0}+\epsilon$. The equilibrium equation provides an explicit expression for $\epsilon$ :

$$
\epsilon=\epsilon\left(l_{0}, m_{L}, m_{u}\right)=2 m_{u} l_{0}\left(3-\frac{m_{L}}{\sqrt{1+4 m_{u}^{2}}}\right)>0
$$

Note that in order to avoid overlapping, the dimensionless parameters must satisfy $m_{u} \in\left(-\frac{1}{2}, \frac{1}{2}\right)$ and $m_{L} \in(0,1)$.

After the linearisation of the system in (38) at the initial condition we obtain a system of DDEs that can be written as, 


$$
\left[\begin{array}{c}
\dot{L_{1}}(t) \\
\dot{L_{2}}(t) \\
\dot{L_{3}}(t)
\end{array}\right]=\left[\begin{array}{ccc}
A & B & C \\
B & A & -C \\
C & -C & D
\end{array}\right]\left[\begin{array}{c}
L_{1}(t-\tau) \\
L_{2}(t-\tau) \\
L_{3}(t-\tau)
\end{array}\right]+\gamma l_{0}\left[\begin{array}{c}
1+\frac{2 m_{L} m_{u}}{3\left(1+4 m_{u}^{2}\right)^{\frac{3}{2}}-m_{L}} \\
1-\frac{2 m_{L} m_{u}}{3\left(1+4 m_{u}^{2}\right)^{\frac{3}{2}}-m_{L}} \\
\frac{1}{\sqrt{1+4 m_{u}^{2}}}\left(1-\frac{4 m_{u}^{2} m_{L}}{3\left(1+4 m_{u}^{2}\right)^{\frac{3}{2}}-m_{L}}\right)
\end{array}\right]
$$

where,

$$
\begin{aligned}
A & =\gamma \frac{m_{L}-2\left(1+4 m_{u}^{2}\right)^{\frac{3}{2}}}{3\left(1+4 m_{u}^{2}\right)^{\frac{3}{2}}-m_{L}} & B & =-\gamma \frac{\left(1+4 m_{u}^{2}\right)^{\frac{3}{2}}}{3\left(1+4 m_{u}^{2}\right)^{\frac{3}{2}}-m_{L}} \\
C & =-\gamma \frac{2 m_{u}\left(1+4 m_{u}^{2}\right)}{3\left(1+4 m_{u}^{2}\right)^{\frac{3}{2}}-m_{L}} & D & =\gamma \frac{m_{L}-\sqrt{1+4 m_{u}^{2}}\left(3+8 m_{u}^{2}\right)}{3\left(1+4 m_{u}^{2}\right)^{\frac{3}{2}}-m_{L}}
\end{aligned}
$$

In order to analyse the stability, we apply a change of variables $\boldsymbol{z}(t):=$ $\boldsymbol{V}^{T} \boldsymbol{L}(t) \in \mathbb{R}^{3}$, where $\boldsymbol{V}$ is the eigenvector matrix of the system above, and use the eigenvalue diagonal matrix

$$
\boldsymbol{D}=\left[\begin{array}{ccc}
-\gamma & 0 & 0 \\
0 & \lambda_{2} & 0 \\
0 & 0 & -\gamma
\end{array}\right]
$$

so that $\lambda_{1}=\lambda_{3}=-\gamma$, and

$$
\lambda_{2}=\gamma \frac{m_{L}-\sqrt{1+4 m_{u}^{2}}\left(3+8 m_{u}^{2}\right)+2\left(1+4 m_{u}^{2}\right)^{\frac{3}{2}}}{3\left(1+4 m_{u}^{2}\right)^{\frac{3}{2}}-m_{L}}<0
$$

The oscillatory regim of the DDE in (39) is then equivalent to the following one,

$$
\dot{\boldsymbol{z}}(t)=\boldsymbol{D} \boldsymbol{z}(t-\tau)
$$

In view of the eigen-values in $\mathbf{D}$, the oscillatory and stability limits of the system are:

$$
\tau_{\text {oscil }}=\frac{1}{e \gamma} \quad \tau_{\text {stabil }}=\frac{\pi}{2 \gamma}
$$

We have also numerically solved the non-linear system in (38) and using a length-dependent delay, different for each segment i.e. $\tau_{i}(t)=\alpha l_{i}(t-$ delta $)$, with $\delta<<1$ the used time-step and $\alpha \in \mathbb{R}_{>0}$. The resulting stability diagram is shown in Figure ?? and resembles the one of the linearised system, but replacing $\tau$ by $\alpha$. 


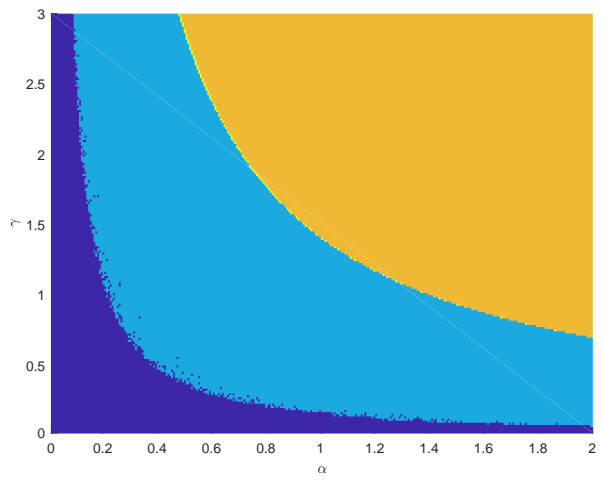

Figure 6: A section of tissue under the displacement $\boldsymbol{u}(t)$-in red-. Edges are enumerated.

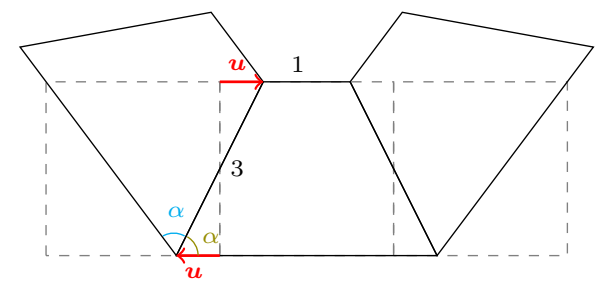

Figure 7: Stability diagram of the cross-section model with on-plane displacements. Dark blue: stable region. Light blue: unstable region. Orange: regions where parameters lead to negative lengths.

\subsection{Out-of-plane deformation}

We consider in this section a strip of tissue where cells are adjacent to each other and a horizontal displacement $\boldsymbol{u}(t)$ acting on the nodes, as shown in Figure 7.

After assuming a constant height and area, the balance equations for each segment yield the following system of equations:

$$
\left\{\begin{array}{l}
\dot{\dot{L}_{1}}(t)=\gamma\left(l_{0}-2 u(t-\tau)-L_{1}(t-\tau)\right) \\
\dot{L_{3}}(t)=\gamma\left(\sqrt{l_{0}^{2}+4 u^{2}(t-\tau)}-L_{3}(t-\tau)\right) \\
0=l_{0}^{2}+12 u^{2}(t)+4 u(t) L_{1}(t)-4 u(t) l_{0}-\sqrt{l_{0}^{2}+4 u^{2}(t)} L_{3}(t)
\end{array}\right.
$$

In order to simplify the subsequent equations, let us define the dimensionless parameters $m_{u}$ and $m_{L}$, and include a small perturbation of $L_{3}(0)$ denoted by $\epsilon$ which guarantees the initial equilibrium:

$$
u(0)=m_{u} l_{0}, \quad L_{1}(0)=m_{L} l_{0}, \quad L_{3}(0)=m_{L} l_{0}+\epsilon,
$$


The equilibrium condition at $t=0$ leads to an expression for $\epsilon$ :

$$
\epsilon=\epsilon\left(l_{0}, m_{L}, m_{u}\right)=l_{0}\left(-m_{L}+\frac{1+12 m_{u}^{2}+4 m_{u} m_{L}-4 m_{u}}{\sqrt{1+4 m_{u}^{2}}}\right)>0
$$

Note that in order to avoid overlapping domains, the paametes must satisfy $m_{u} \in\left(-\frac{1}{2}, \frac{1}{2}\right)$ and $m_{L} \in(0,1)$.

\subsubsection{Analysis of linearised form}

The linearisation of (40) yields the following system of DDEs

$$
\left\{\begin{array}{c}
\dot{L_{1}}(t)=\gamma\left(l_{0}-2 u(t-\tau)-L_{1}(t-\tau)\right) \\
\dot{L_{3}}(t)=\gamma\left(\frac{l_{0}^{2}}{\sqrt{l_{0}^{2}+4 u_{0}^{2}}}-L_{3}(t-\tau)+\frac{4 u_{0}}{\sqrt{l_{0}^{2}+4 u_{0}^{2}}} u(t-\tau)\right) \\
4 u_{0}\left(L_{1}(t)-L_{10}-3 u_{0}-6 u(t)+\frac{L_{30}\left(u_{0}-u(t)\right)}{\sqrt{l_{0}^{2}+4 u_{0}^{2}}}\right) \\
=\sqrt{l_{0}^{2}+4 u_{0}^{2}} L_{3}(t)+\left(4 l_{0}-4 L_{10}\right) u(t)-l_{0}^{2}
\end{array}\right.
$$

Note that from the coefficient of $u(t)$ in the equilibrium equation is zero when $m_{L}=-12 m_{u}^{3}-5 m_{u}+1$. Using the last equations, the system of equations above can be reduced to,

$$
\left\{\begin{array}{c}
\dot{L_{1}}(t) \\
\dot{L_{3}}(t)
\end{array}\right\}=\left[\begin{array}{cc}
A & B \\
C & D
\end{array}\right]\left\{\begin{array}{l}
L_{1}(t-\tau) \\
L_{3}(t-\tau)
\end{array}\right\}+\gamma \boldsymbol{b}
$$

with

$$
\begin{aligned}
& \boldsymbol{b}=\left\{\begin{array}{c}
l_{0}-\frac{2\left(l_{0}^{2}-12 u_{0}^{2}-4 u_{0} L_{10}+\frac{4 u_{0}^{2} L_{30}}{\sqrt{l_{0}^{2}+4 u_{0}^{2}}}\right)}{-\frac{4 u_{0} L_{30}}{\sqrt{l_{0}^{2}+4 u_{0}^{2}}}-4 l_{0}+4 L_{10}+24 u_{0}} \\
\frac{4 u_{0}\left(l_{0}^{2}-12 u_{0}^{2}-4 u_{0} L_{10}+\frac{4 u_{0}^{2} L_{30}}{\sqrt{l_{0}^{2}+4 u_{0}^{2}}}\right)}{\sqrt{l_{0}^{2}+4 u_{0}^{2}}}+\frac{\sqrt{l_{0}^{2}+4 u_{0}^{2}}\left(-\frac{4 u_{0} L_{30}}{\sqrt{l_{0}^{2}+4 u_{0}^{2}}}-4 l_{0}+4 L_{10}+24 u_{0}\right)}{l^{2}}
\end{array}\right\} \\
& A=-\gamma+\frac{8 \gamma u_{0}}{-\frac{4 u_{0} L_{30}}{\sqrt{l_{0}^{2}+4 u_{0}^{2}}}-4 l_{0}+4 L_{10}+24 u_{0}} \quad B=\frac{-2 \gamma \sqrt{l_{0}^{2}+4 u_{0}^{2}}}{-\frac{4 u_{0} L_{30}}{\sqrt{l_{0}^{2}+4 u_{0}^{2}}}-4 l_{0}+4 L_{10}+24 u_{0}} \\
& C=\frac{-\left(\frac{16 \gamma u_{0}^{2}}{\sqrt{l_{0}^{2}+4 u_{0}^{2}}}\right)}{-\frac{4 u_{0} L_{30}}{\sqrt{l_{0}^{2}+4 u_{0}^{2}}}-4 l_{0}+4 L_{10}+24 u_{0}} \quad D=-\gamma+\frac{4 \gamma u_{0}}{-\frac{4 u_{0} L_{30}}{\sqrt{l_{0}^{2}+4 u_{0}^{2}}}-4 l_{0}+4 L_{10}+24 u_{0}}
\end{aligned}
$$

The eigenvalues of the system matrix are,

$$
\lambda_{1}=-\gamma<0, \quad \lambda_{2}=\gamma\left(\frac{-2 m_{u}-m_{L}+1}{12 m_{u}^{3}+5 m_{u}+m_{L}-1}\right)
$$


These expression allows us to define four different stability and oscillatory regions according to the sign and values of $\lambda_{2}$, as shown in Figure 8:

- In Regions I, II:

$$
\tau_{\text {oscil }}=\frac{1}{e \gamma}, \quad \tau_{\text {stabil }}=\frac{\pi}{2 \gamma}
$$

- In Region III:

$$
\tau_{\text {oscil }}=\frac{12 m_{u}^{3}+5 m_{u}+m_{L}-1}{e \gamma\left(2 m_{u}+m_{L}-1\right)}, \quad \tau_{\text {stabil }}=\frac{\pi\left(12 m_{u}^{3}+5 m_{u}+m_{L}-1\right)}{2 \gamma\left(2 m_{u}+m_{L}-1\right)}
$$

- In Region IV, the system is always unstable.

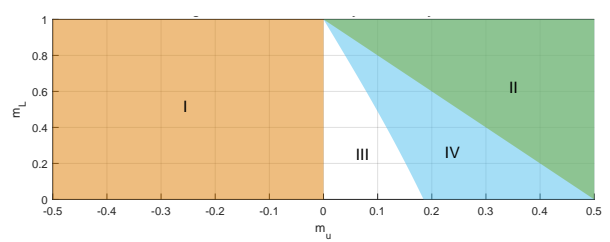

Figure 8: Stability regions according to sign and values of $\lambda_{1}$ and $\lambda_{2}$ for outof-plane deformation of cross-section. $\lambda_{1}$ defines regions $I$ and $I I . \quad \lambda_{2}$ defines region $I I I$. In region $I V, \lambda_{2}>0$ and the system is unstable.

\subsubsection{Stability of non-linear form}

Similarly to Section 2.4, we note that the non-linear system in (40) admits the solution $\dot{u}=0$ when $\gamma$ is uniform, unlike the linear system in (38). This is however not true for the linear system. To see this, the equilibrium equation of the linear system is:

$$
\sum_{i} k\left(l_{i}-L_{i}(t)\right) c_{i}+b_{0}=0, \quad c_{i}=\frac{\partial l_{i}}{\partial u} \text { and } \dot{l}_{i}(t)=0,
$$

where $b_{0}\left(m_{u}, m_{L}\right) \neq 0$. Differentiation of the first equation leads to a contradictory results:

$$
\sum_{i} k \dot{L}_{i}(t) c_{i}=0 \Rightarrow \sum_{i} k \gamma\left(l_{i}-L_{i}(t)\right) c_{i}=0 \quad \forall t \Rightarrow b_{0}=0
$$

showing that the linear system does not admit $u=u_{0}$. When $u=u_{0}$, the DDE remain unchanged and uncouple, and thus the stability bounds are those of a single element, i.e. $\tau_{\text {stab }}<\pi /(2 \gamma)$. 


\section{Conclusions}

We have analysed the stability and the presence of oscillatory solutions of a simplified planar deformation mode in a regular homogeneous epithelium, represented by a three-bar system, and also a simplified cross-section of the tissue. Resorting to a rheological law with variable rest-length $[4,8]$ and a delayed response $[16,17]$, we have deduced stability and oscillatory bounds, which depend on the remodelling rate $\gamma$ and the delay $\tau$. For increasing values of the remodelling rate, the tissues exhibits a lower viscous response, resulting in a more oscillatory or unstable dynamics of the rest-length.

We have demonstrated that linear stability analysis may restrict the bounds, introducing a dependence on the initial conditions for some initial stressed states. This dependence is in fact absent when considering the non-linear version of the equilibrium equations, as our numerical solutions also illustrate.

Although we have not given general stability conditions for the non-linear system, we have been able to give some results for some general initial conditions. The bounds are in fact identical to those deduced in linear unidimensional analysis [17].

The stability and origin of the oscillatory deformation of cells is now under intensive investigation [24, 20]. Although experimentalists have detected and measured delays between the concentration of motor proteins (myosin or cadherin) [7], the relation between these oscillations and eventual drastic tissue deformations remains elusive. Although the work presented here aims at suggesting potential causes behind the observed instabilities, further measurements are needed to corroborate the deduced instability and oscillatory regimes.

\section{Acknowledgements}

J.J.M. and M.A.D. are financially supported by the Spanish Ministry of Science, Innovation and Universities (MICINN) through grant DPI2016-74929-R, while C.B. has been supported by the Maria de Maetzu programme of MICINN at Barcelona Graduate School of Mathematics (BSGMath) through grant MdM2014-0445. J.J.M has been also financed by government of Catalonia through grant 2017 SGR 1278.

\section{References}

[1] Asl, F.M., Ulsoy, A.G.: Analysis of a system of linear delay differential equations. J. Dyn. Sys. Meas. Contr. 125, 215-223 (2003)

[2] Cavanaugh, K., Staddon, M., Munro1, E., Banerjee, S., Gardel, M.: RhoA mediates epithelial cell shape changes via mechanosensitive endocytosis. Biorxiv (2019). Http://dx.doi.org/10.1101/605485 
[3] Chi, H., Bell, J., Hassard, B.: Numerical solution of a nonlinear advancedelay-differential equation from nerve conduction theory. J. Math. Biol. 24, 583-601 (1986)

[4] Clément, R., Collinet, C., Dehapiot, B., Lecuit, T., Lenne, P.: Viscoelastic dissipation stabilizes cell shape changes during tissue morphogenesis. Current Biol. 27(20), 3132-3142 (2017)

[5] Cooke, K., Turi, J.: Stability, instability in delay equations modeling human respiration. J. Math. Biol. 32(6), 535-543 (1994)

[6] Corless, R., Gonnet, G., Hare, D., Jeffrey, D., Knuth, D.: On the Lambert W function. Adv. Comp. Math. 5, 329-359 (1996)

[7] Dierkes, K., Sumi, A., Solon, J., Salbreux, G.: Spontaneous Oscillations of Elastic Contractile Materials with Turnover. Phys. Rev. Letters 113, $148102(2014)$

[8] Doubrovinski, K., Swan, M., Polyakov, O., Wieschaus, E.: Measurement of cortical elasticity in drosophila melanogaster embryos using ferrofluids. Proc. Natl. Acad. Sci. USA 114(5), 1051-1056 (2017)

[9] Erneux, T.: Applied Delay Differential Equations, Surveys and Tutorials in the Applied Mathematical Sciences, vol. 3. Springer, New York (2009)

[10] Getto, P., Gyllenberg, M., Nakata, Y., Scarabel, F.: Stability analysis of a state-dependent delay differential equation for cell maturation: analytical and numerical methods. J. Math. Biol. 79, 281-328 (2019)

[11] Kaouri, K., Maini, P.K., Skourides, P.A., Christodoulou, N., Chapman, S.J.: A simple mechanochemical model for calcium signalling in embryonic epithelial cells. J. Math. Biol. 78, 2059-2092 (2019)

[12] Khalilgharibi, N., Fouchard, J., Asadipour, N., Barrientos, R., Duda, M., Bonfanti, A., Yonis, A., Harris, A., Mosaffa, P., Fujita, Y., Kabla, A., Mao, Y., Baum, B., Muñoz, J., Miodownik, M., Charras, G.: Stress relaxation in epithelial monolayers is ocntrolled by actomyosin cortex. Nature Phys. (2019). DOI 10.1038/s41567-019-0516-6. Accepted

[13] Lapytsko, A., Schaber, J.: The role of time delay in adaptive cellular negative feedback systems. J. Theor. Biol. 308, 64-73 (2016)

[14] Martin, A., Kaschube, M., Wieschaus, E.: Pulsed contractions of an actinmyosin network drive apical constriction. Nature 457, 495-499 (2009)

[15] Munjal, A., Philippe, J., Munro, E., Lecuit, T.: A self-organized biomechanical network drives shape changes during tissue morphogenesis. Nature 524(7565), 351-355 (2015)

[16] Muñoz, J., Albo, S.: Physiology-based model of cell viscoelasticity. Phys. Rev. E 88(1), 012708 (2013) 
[17] Muñoz, J., Dingle, M., Wenzel, M.: Mechanical oscillations in biological tissues as a result of delayed rest-length changes. Phys. Rev. E 98(1), 052409 (2018)

[18] Notbohm, J., Banerjee, S., Utuje, K., Gweon, B., amd Y. Park, H.J., Shin, J., Butler, J., Fredberg, J., Marchetti, M.: Cellular contraction and polarization drive collective cellular motion. Bioph. J. 110(12), 2729-2738 (2012). DOI 10.1016/j.bpj.2016.05.019

[19] Oosterwyck, H.: Computational mechanobiology: may the force be with you. J. Math. Biol. 70(6), 1323-1326 (2015)

[20] Petrolli, V., Goff, M., Tadrous, M., Martens, K., Allier, C., Mandula, O., Hervé, L., Henkes, S., Sknepnek, R., Boudou, T., Cappello, G., Balland, M.: Confi

nement-induced transition between wave-like collective cell migration modes. BioRxiv (2018). DOI dx.doi.org/10.1101/495747. Https://www.biorxiv.org/content/10.1101/495747v1

[21] Peyret, G., Mueller, R., d'Alessandro, J., Begnaud, S., Marcq, P., Mège, R., Yeomans, J., Doostmohammadi, A., Ladoux, B.: Sustained oscillations of epithelial cell sheets. BioRxiv (2018). DOI dx.doi.org/10.1101/492082. Https://www.biorxiv.org/content/10.1101/492082v1

[22] Roldán, L., Muñoz, J., Sáez, P.: Computational modeling of epithelial wound healing: Short and long term chemo-mechanical mechanisms. Comp. Meth. Appl. Mech. Engng. 350, 25-56 (2019)

[23] Shinozaki, H., Mori, T.: Robust stability analysis of linear time-delay systems by Lambert W function: Some extreme point results. Automat. 42(1), 1791-1799 (2006)

[24] Solon, J., Kaya-Copur, A., Brunner, D.: Pulsed forces timed by a ratchetlike mechanism drive directed tissue movement during dorsal closure. Cell 58(137), 1331-1342 (2009)

[25] Staddon, M., Cavanaugh, K., Munro, E., Gardel, M., Banerjee, S.: Mechanosensitive junction remodelling promotes robust epithelial morphogenesis. BioRxiv (2019)

[26] Sumi, A., Hayes, P., D’Angelo, A., Salbreux, J.C.G., Dierkes, K., Solon, J.: Adherens junction length during tissue contraction is controlled by the mechanosensitive activity of actomyosin and junctional recycling. Dev. Cell 47(4), 453-463 (2018)

[27] Yi, S., Nelson, P., Ulsoy, A.: Delay differential equations via the matrix Lambert $\mathrm{W}$ function and bifurcation analysis: Application to machine tool chatter. Math. Biosc. Engin. 4(2), 255-369 (2007) 